\title{
Can maternally inherited endosymbionts adapt to a novel host? Direct costs of Spiroplasma infection, but not vertical transmission efficiency, evolve rapidly after horizontal transfer into D. melanogaster
}

\author{
S Nakayama ${ }^{1}$, SR Parratt ${ }^{2}$, KJ Hutchence, Z Lewis, TAR Price and GDD Hurst \\ Maternally inherited symbionts are common in arthropods and many have important roles in host adaptation. The observation that \\ specific symbiont lineages infect distantly related host species implies new interactions are commonly established by lateral \\ transfer events. However, studies have shown that symbionts often perform poorly in novel hosts. We hypothesized selection on the \\ symbiont may be sufficiently rapid that poor performance in a novel host environment is rapidly ameliorated, permitting symbiont \\ maintenance. Here, we test this prediction for a Spiroplasma strain transinfected into the novel host Drosophila melanogaster. In \\ the generations immediately following transinfection, the symbiont had low transmission efficiency to offspring and imposed severe \\ fitness costs on its host. We observed that effects on host fitness evolved rapidly, being undetectable after 17 generations in the \\ novel host, whereas vertical transmission efficiency was poorly responsive over this period. Our results suggest that long-term \\ symbiosis may more readily be established in cases where symbionts perform poorly in just one aspect of symbiosis.
}

Heredity (2015) 114, 539-543; doi:10.1038/hdy.2014.112; published online 4 February 2015

\section{INTRODUCTION}

Estimates suggest that above $70 \%$ of all arthropods harbour nonobligate, 'secondary' endosymbionts within their cells (Duron et al., 2008; Zug et al., 2012). In many cases, symbionts represent an important component of 'host adaptation to environment', conferring important properties such as resistance and tolerance to natural enemies (reviewed in Haine 2008; Brownlie and Johnson, 2009). Despite being important in host adaptation, recent molecular analyses of associations between secondary symbionts and their hosts provide evidence of successful and frequent interspecific transmission of symbionts throughout evolutionary time, alongside multiple loss events (Pool et al., 2006; Haselkorn et al., 2009; Raychoudhury et al., 2009; Mouton et al., 2012). The gain of function that can occur on symbiont acquisition and the frequency with which this occurs in nature, has led to them being considered akin to plasmids in representing heritable genomic components that can be acquired by a host species and alter its biology (Jiggins and Hurst, 2011).

It has been observed that symbionts commonly perform poorly immediately following a host-shift event. For example, weak vertical transmission efficiency after a host shift has been noted in a number of examples (for example, Sakaguchi and Poulson, 1963; Clancy and Hoffmann, 1997; Russell and Moran, 2005; Kageyama et al., 2006; Tinsley and Majerus, 2007). Significant direct costs in host longevity, fecundity and egg-hatch rates have been also reported following lateral transfer of Wolbachia into a novel Drosophila host species (McGraw et al., 2002; Carrington et al., 2010), and Spiroplasma into novel ladybird species (Tinsley and Majerus, 2007).

The 'misfit' observed following host-shift events raises the question as to the processes involved in the initial establishment of a symbiont. One possibility is that many transfer events are occurring, and a fraction of these produce very well-fitted symbioses, and it is these that persist. In support of this, some new symbiont/host combinations do function well, and are predicted to drive through populations should transfer occur (Haselkorn et al., 2013). A second, and not mutually exclusive hypothesis, is that rapid adaptation of symbionts to novel host environments permits long-term persistence of symbionts whose initial performance in a host is poor. Indeed, several studies have shown attenuated direct costs to the fitness of the host over time in the virulent 'popcorn' strain of Wolbachia (McGraw et al., 2002; Carrington et al., 2010), as well as evolution towards mutualism of strains in nature (Weeks et al., 2007).

The genus Spiroplasma is one of the most common maternally inherited endosymbiont groups, with a wide range of hosts including insects, crustaceans, arachnids and plants (Gasparich et al., 2004). The associations between Spiroplasma and their hosts range from parasitism and reproductive manipulation (Counce and Poulson, 1962; Williamson and Poulson, 1979; Williamson et al., 1999) to mutualism (Jaenike et al., 2010; Xie et al., 2010). In Drosophila hydei, Spiroplasma shows very high vertical transmission rates, and provides protection to its host against parasitic wasp attack (Xie et al., 2010). Importantly,

Evolution, Ecology and Behaviour, Institute of Integrative Biology, University of Liverpool, Liverpool, UK

${ }^{1}$ Current address: Department of Biology, Graduate School of Science, Kyushu University, 6-10-1, Hakozaki, Higashi-ku, Fukuoka 812-0053, Japan.

${ }^{2}$ Current address: Metapopulation Research Group, Department of Biosciences, University of Helsinki, Helsinki, Finland.

Correspondence: Dr S Nakayama, Department of Biology, Graduate School of Science, Kyushu University, 6-10-1, Hakozaki, Higashi-ku, Fukuoka 812-0053, Japan.

E-mail: nakayama58611@gmail.com

Received 25 April 2014; revised 23 October 2014; accepted 27 October 2014; published online 4 February 2015 
Macrocheles body mites on $D$. hydei were observed to carry Spiroplasma (Osaka et al., 2013), and Spiroplasma can be transmitted into D. melanogaster via ectoparasitic mites (Jaenike et al., 2007). Further, D. melanogaster naturally host other Spiroplasma strains in wild populations (Montenegro et al., 2005). Thus, the symbiont has the potential to regularly undergo host-shifts in nature, and D. melanogaster is a promising host for Spiroplasma infection. However, previous work has indicated that the Spiroplasma from $D$. hydei misfits in $D$. melanogaster, exhibiting virulence and low vertical transmission efficiency (Kageyama et al., 2006). This disturbance is also observed in greater perturbation of host transcriptional patterns in transinfected D. melanogaster compared with naturally infected controls (Hutchence et al., 2011).

To date, studies of evolution post artificial transfer have focused on strains that misfit in either vertical transmission or in producing virulence in the novel host. The focus of this study is to clarify whether a misfit of symbiont-host association just after a host-shift event can be ameliorated in terms of the symbiont's adaptation, and if so how rapidly such an adaptation can occur. We thus artificially transfer a strain of Spiroplasma from its native host, D. hydei, to a novel host, D. melanogaster, and investigate whether both vertical transmission efficiency increases, and direct costs to host fitness are ameliorated, by selection following a host-shift event.

\section{MATERIALS AND METHODS}

\section{Symbiont and host}

Spiroplasma infecting D. hydei (Spiroplasma strain TEN 104-106 haplotype 1, HY1 or 'ancestral' Spiroplasma hereafter, Mateos et al., 2006) was used in this study. HY1 falls as an out-group of Spiroplasma poulsonii, and shows no evidence of reproductive manipulation in the Canton-S (CS hereafter) strain of D. melanogaster, which was used as a novel fly host (Hutchence, 2011).

\section{Artificial transfer of Spiroplasma into D. melanogaster}

An outline of our experiments in this study is provided in Figure 1. First, we artificially infected flies with Spiroplasma using a hydraulic positive-pressure microinjection apparatus (Model IM-6, Narushige Ltd, Tokyo, Japan). To this end, $0.1-0.2 \mu \mathrm{l}$ of haemolymph was extracted from the thorax of HY1-infected $D$. hydei females by capillary action, and was injected into the abdomen of virgin CS females. Each injected female was then left for 14 days to give the Spiroplasma time to establish, and was then placed with two CS males in a vial containing $15 \mathrm{ml}$ standard Drosophila medium (oats, sugar, water, yeast, agar and nipagin). Two males were introduced to ensure mating success. Females were allowed to oviposit for 4 days. The females were then screened for Spiroplasma by the PCR assay, and any uninfected mothers and their offspring were discarded. PCR assays were conducted as follows. Each female was macerated in a $50 \mu \mathrm{l} 5 \%$ Chelex (Chelex 100 Resin, Bio-Rad Laboratories, Hercules, CA, USA) solution and $1 \mu \mathrm{l}$ proteinase $\mathrm{K}$, and incubated at $37^{\circ} \mathrm{C}$ overnight (Walsh et al., 1991). Samples were then heated at $95^{\circ} \mathrm{C}$ for $10 \mathrm{~min}$ to denature the proteinase K. PCR amplifications were performed using Spiroplasma-specific primers SpoulF (5'-GCTTAACTCCAGTTCGCC-3') and SpoulR (5'-CCTGTCTCAATGTTAACCTC-3') as in Montenegro et al. (2005). PCR cycling conditions were an initial denature of $1 \mathrm{~min} 30 \mathrm{~s}$ at $94^{\circ} \mathrm{C}$, followed by 35 cycles of $15 \mathrm{~s}$ at $93^{\circ} \mathrm{C}, 1 \mathrm{~min}$ annealing at $47^{\circ} \mathrm{C}$ and $1 \mathrm{~min}$ at $72^{\circ} \mathrm{C}$. These infected females were designated as the parental generation.

\section{Establishment and maintenance of Spiroplasma in the novel host, D. melanogaster}

From the offspring of four of the successfully Spiroplasma-infected parental females, five virgin daughters were randomly selected as the F1 generation for the selection lines. These F1 females were aged for 4 days, placed individually in a standard food vial with two CS males for mating and allowed to oviposit for 4 days. Afterwards, all F1 females were screened for Spiroplasma with the diagnostic PCR described above, and uninfected individuals were discarded along with the vials in which they had oviposited. When offspring emerged from the infected vials 10 days post oviposition, all virgin females were collected and thoroughly mixed, and 30 were randomly selected as mothers of the next generation; this enabled selection on both cost of infection and vertical transmission efficiency. These virgin females were aged for 4 days, and the procedure of oviposition, screening and selection were repeated as above. This procedure was continued every 14 days for 25 generations with four replicate populations (see Supplementary Table 1 for the sample size at each generation). Vertical transmission efficiencies of Spiroplasma were calculated using the number of infected and uninfected mothers at each host generation, in the knowledge that all individuals had infected mothers (as uninfected mothers and their offspring were discarded from each generation). Quality control for PCR assays included (a) a positive control re-extracted alongside the test individuals (D. melanogaster carring Melanogaster Sex Ratio Organism: MSRO) and (b) repeat PCR assays of negative specimens.

\section{Measurement of fitness}

To measure the impact of Spiroplasma on the fitness of the novel host, we measured the number of offspring produced by an infected female. However,

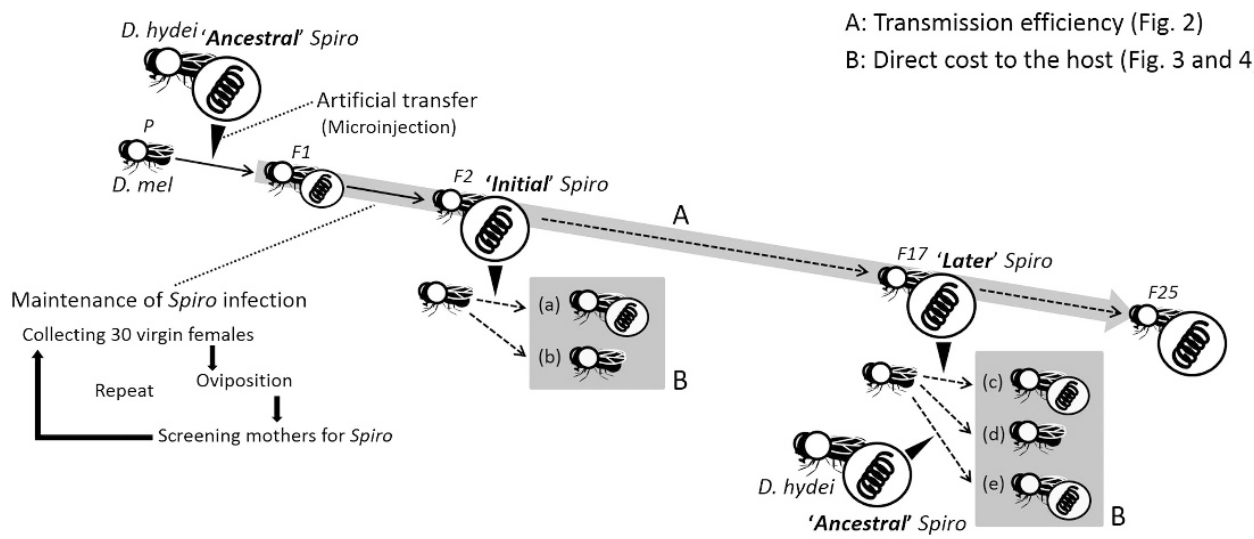

Figure 1 Outline of experimental design. Following transinfection, four Spiroplasma-infected lines were obtained. In each line, 30 females were permitted to lay each generation, and following reproduction, assayed for the Spiroplasma. The progeny of infected females were then pooled to found the next generation, and the process iterated to create F17 of passage. Transmission efficiency was measured each generation as the fraction of the 30 females carrying the Spiroplasma. Fitness of flies carrying Spiroplasma in each replicate was measured at generation 2 ('initial': a) compared with CS controls (b). Fitness was measured again at F17. In this case, the passaged 'later' Spiroplasma strains was transinfected across to an unexposed CS line (c) alongside a Spiroplasma strain from D. hydei ('ancestral': e), and the fitness of females of these measured alongside a CS control (d). All recipients of $D$. melanogaster and donors of $D$. hydei for artificial transfer of Spiroplasma were provided from stock culture in laboratory. See the text for a more detailed protocol. 
transinfection may itself be associated with transient changes in the properties of a symbiosis, making it unfair to test newly transfected lines with Spiroplasma in the selected lines. To avoid this, we transinfected the Spiroplasma from the selected lines into the CS stock line and then measured fitness, such that all fitness measures were comparable. This measurement of fitness following transinfection also enables us to exclude any possibility of evolution of the host, D. melanogaster. Haemolymph was extracted from females of the selected lines of D. melanogaster, and was injected into virgin CS females from the stock population. These injected females were left for 14 days, as described previously, and then placed in a vial with two CS males. After 4 days, those females (18-25 females per line) were screened for Spiroplasma, and offspring were raised from successfully infected mothers. Collected daughters were thoroughly mixed before being randomly selected, and left for 4 days to sexually mature, after which each female was placed with two CS males in a small plastic vial $(2 \mathrm{~cm}$ height $\times 2 \mathrm{~cm}$ diameter $)$ containing grape jelly that facilitates larval collection. After 2 days, the females were screened for Spiroplasma and first-instar larvae were collected only from the offspring of infected mothers. Collected larvae were put in standard food vials at a density of 30 larvae per vial. Eclosed virgin females from those vials were used for the fitness measurement ('tester females'). Tester females were aged for 7-10 days and then put with two CS males in a vial with fly medium for mating. They were moved into fresh vials every day for 5 days to allow continuous oviposition. After the fifth day, females were screened for Spiroplasma, and vials that been occupied by uninfected females were discarded. Eclosed offspring from the second-, third-, fourth- and fifth-day vials were counted as an index of the direct influence of Spiroplasma on host fitness. Spiroplasma living in the F2 ('initial') and F17 ('later') generations of the selected lines were used for the measurement. Female flies are often sensitive to the season and weather; for example, they lay fewer eggs when it is raining, possibly indicated by pressure changes that were beyond our control. Therefore to ensure our conclusions were robust despite fitness measurements being conducted on different dates, control treatments were simultaneously performed for both measurements ('initial' and 'later' Spiroplasma). We used uninfected CS females in the same way as described above, but without the microinjection and PCR assays. These controls enabled us to compare results for 'initial' and 'later' Spiroplasma (see Results). In the experiment using the 'later' Spiroplasma, additional CS females were infected with 'ancestral' Spiroplasma (that is, HY1 taken directly from $D$. hydei) and the offspring production of their infected granddaughters was examined as above concurrently with the 'later' strains to allow direct comparison of fitness effects between novel and evolved strains. Finally, for both generations the relative fitness of Spiroplasma-infected females to uninfected controls was calculated as the ratio of the number of offspring produced by Spiroplasma-infected females over 4 days, to that of uninfected control females.

\section{Statistical analyses}

Generalized linear models with logit links were used for the analyses of change in transmission efficiency (ratio data). The perfect transmission efficiency of Spiroplasma was observed in the F1 generation in all replicates, but afterwards, it kept decreasing in the F2 and F3 generations (see Figure 1). Kageyama et al. (2006) reported a similar pattern, whereby the transmission efficiency of HY1 Spiroplasma was high immediately following transinfection from $D$. hydei into D. melanogaster, but disappeared by the third generation. Although the reason for this pattern is as yet unknown, our data suggest that the drastic decline in HY1 Spiroplasma infection immediately following transinfection into D. melanogaster may always occur, regardless of the presence or absence of directional selection for transmission efficiency. Here we are interested in whether poor transmission efficiency of Spiroplasma after transinfection increases via selection. We therefore decided to exclude the data in the F1, F2 and F3 generations from the analyses, which consistently showed a persistent decline in transmission in all replicates.

The number of offspring produced by the CS females having different infection statuses was analyzed using general linear models. Selection replicates were nested within the infection status in the analyses. Differences in the mean relative fitness of females infected with the 'initial' and 'later' Spiroplasma were also tested using paired $t$-tests. All statistics was performed using JMP version 7.0 (SAS Institute, 2009).

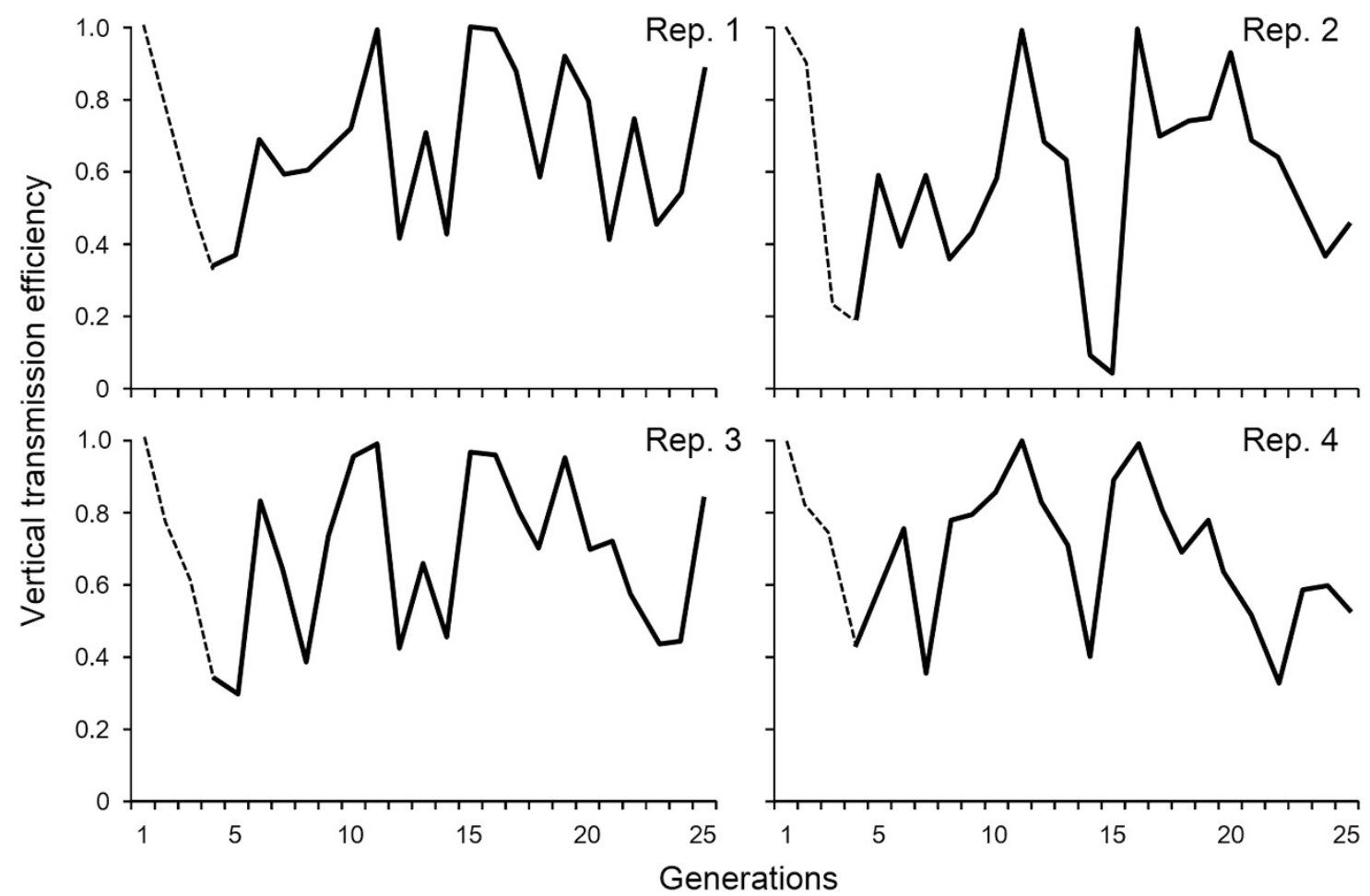

Figure 2 Vertical transmission efficiency of Spiroplasma following lateral transfer to $D$. melanogaster. Significant regression coefficients were observed between the data from the F4 to F25 generations (solid lines) in replicates 1, 2, and 3, but not in replicate 4 (see text for details). Dashed lines show the data between the first three generations, which was not included in the analyses (see text for details). 


\section{RESULTS}

Vertical transmission efficiency

Figure 2 shows the changes in vertical transmission efficiency of Spiroplasma after artificial lateral transfer into D. melanogaster. Because the model showed a significant interaction between generation and replicate $\left(\chi^{2}=17.96, P<0.001\right)$, analyses were performed for each replicate lineage with sequential Bonferroni correction (Rice, 1989). Although the transmission rates fluctuated markedly in all replicates, three of the four replicates showed significant increases in transmission efficiency, with very small regression coefficients (replicate 1, $r=0.040 \pm 0.013$ s.e., $\chi^{2}=8.92, P=0.0028$; replicate $2, r=0.053 \pm$ $0.012, \chi^{2}=16.15, P<0.0001$; replicate $3, r=0.035 \pm 0.013, \chi^{2}=6.57$, $P=0.0104$; replicate $\left.4, r=-0.021 \pm 0.013, \chi^{2}=2.54, P=0.110\right)$

\section{Direct cost on host fitness}

D. melanogaster females infected with 'initial' Spiroplasma produced fewer offspring than uninfected ones $\left(F_{1,72}=17.6, \quad P<0.0001\right.$; Figure 3$)$. There was no significant effect of replicate $\left(F_{3,72}=1.98\right.$, $P=0.123)$. In contrast, we found no significant difference between

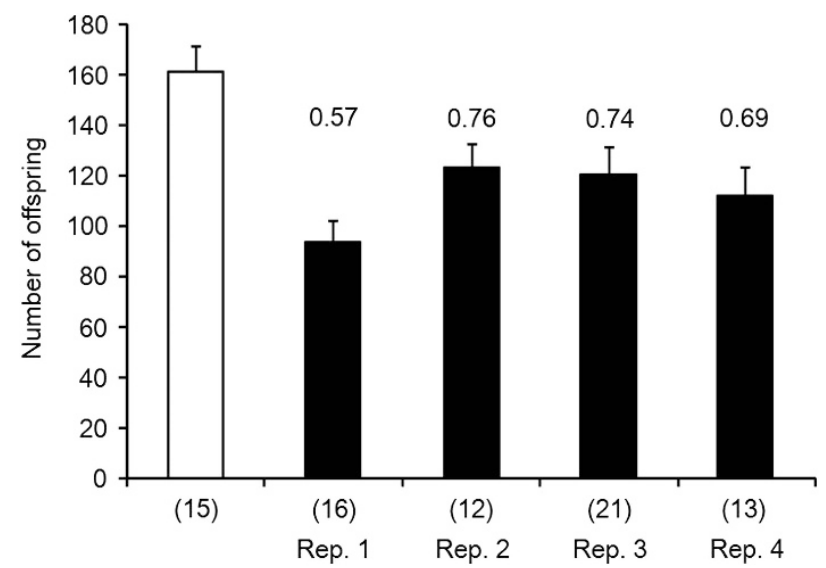

Figure 3 Mean number of offspring produced over 4 days by $D$. melanogaster females infected with Spiroplasma derived from the F2 generation (four selected lines, filled bars) and uninfected control (open bar). Values on bars and numbers in parentheses show fitness relative to control and sample size, respectively; error bar represents one s.e.m.

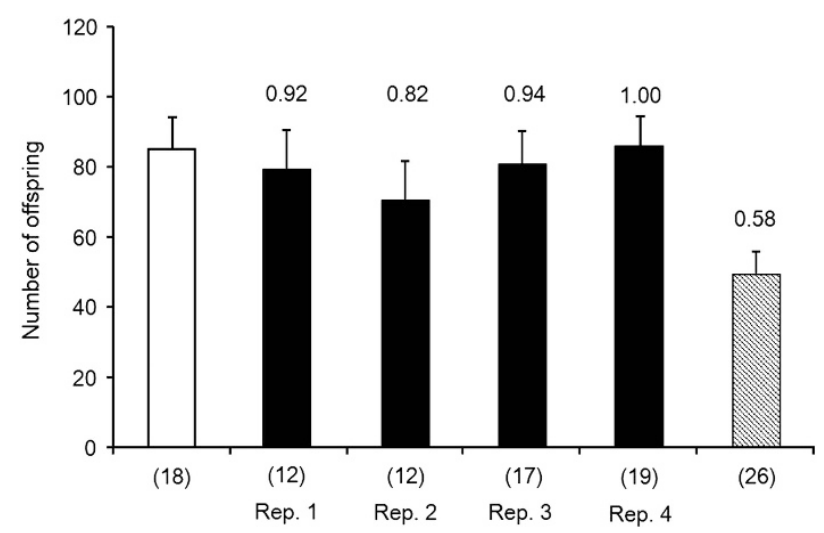

Figure 4 Mean number of offspring produced over 4 days by $D$. melanogaster females infected with Spiroplasma derived from the F17 generation (four selected lines, filled bars), ancestral Spiroplasma taken from D. hydei (diagonal bar) and uninfected control (open bar). Values on bars and numbers in parentheses show fitness relative to control and sample size, respectively; error bar represents one s.e.m. family sizes of females infected with 'later' Spiroplasma and uninfected control flies $\left(F_{1,73}=0.353, P=0.553\right.$; Figure 4$)$. Again, there was no effect of replicate $\left(F_{3,73}=0.382, P=0.766\right)$. Females infected with 'later' Spiroplasma (infection status, $F_{1,81}=13.63, P<0.001$; selection replicates, $F_{3,81}=0.545, P=0.652$ ) and uninfected control females $\left(F_{1,42}=8.00, P=0.007\right)$ both produced a significantly greater number of offspring than those infected with concurrently measured 'ancestral' Spiroplasma (Figure 4). Mean relative fitness of females infected with Spiroplasma significantly increased between the F2 (relative fitness to controls: $0.69 \pm 0.04$ s.e. $)$ and F17 $(0.92 \pm 0.04$ s.e. $)$ generations $(t=3.77, P=0.032$; see Figures 3 and 4 for values in each line).

\section{DISCUSSION}

The rate of establishment of symbionts into previously uninfected host species is an important component of their commonness in nature. Theory suggests that after transfer to a novel host, endosymbionts should be strongly selected to increase their transmission rate to offspring, and to reduce the harm to their host (Jaenike, 2012). This process, combined with sufficient drive in the form of natural enemy resistance or reproductive manipulation, could potentially permit long-term persistence of initially unbalanced symbioses created following introduction to a novel host species.

In this study, we examined the trajectory of evolution for a symbiont that performed poorly both in terms of virulence and vertical transmission in its novel host. We found that although vertical transmission efficiency of Spiroplasma increased only slowly over time, attenuation of its direct cost to host fitness was much more pronounced: an initially virulent symbiont evolved to be benign after 17 generations. Importantly, the impact of the passaged Spiroplasma ('later') was compared concurrently with one recently transinfected into the host ('ancestral'), which would mimic the properties of the 'initial' strain (see Figure 4). In these assays, the passaged Spiroplasma had a lower effect on host fecundity than a strain recently exposed to the host, and the relative effect of the newly transinfected strain, compared with the control, was similar to that observed in the previous assay. Moreover, because the fitness measurements were conducted following transinfection of each type of Spiroplasma into the uninfected CS strain as described earlier, the result can be attributed to change in the Spiroplasma infection, but not evolution of the host.

The vertical transmission data indicated that the mother-offspring passage was inefficient compared with native infections, transmission efficiency was quite variable between generations and that although improvements over passage were observed, these were minor and insufficient to permit Spiroplasma maintenance in the novel host. Segregational loss during vertical transmission is typically observed in transinfection between distantly related host species, and reflects maladaptation of the symbiont (Clancy and Hoffmann, 1997; Kageyama et al., 2006; Hutchence et al., 2011). The variability of vertical transmission we observed reflects that previously observed for our system on a more limited data set (Kageyama et al., 2006). The reasons for variability of transmission have not been considered extensively previously, but we believe it reflects the same maladaptation in transmission biology. Importantly, poor vertical transmission in this system is not primarily associated with reduced titre, with Spiroplasma from $D$. hydei achieving a titre in $D$. melanogaster equivalent to strains native to $D$. melanogaster (Kageyama et al., 2006). This equality of titre (contrasting with lack of equality of transmission) suggests that it is the process of establishment in eggs from the soma that impedes vertical transmission. We hypothesize that the failure of the Spiroplasma to respond to selection for increased vertical transmission is because of a lack of genetic variation available to promote the fit between the microbe and novel host for 
movement into the germ line within the time frame and population size of our experiment. For Spiroplasma, movement to the germ line involves binding to egg-yolk proteins to achieve transit into the developing oocyst (Herren et al., 2013). One possibility is that egg-yolk proteins diverge among species, producing a misfit of the vertical transmission mechanism, and selection must rectify this misfit.

The vertical transmission efficiency data contrast with the rapid evolution towards benign impacts of Spiroplasma infection on D. melanogaster fecundity, in which its strong pathogenicity was observed to be undetectable after 17 host generations. Titre alone is a poor explanation for pathogenicity in this system, as the introduced Spiroplasma does not reach a higher titre than native strains (Kageyama et al., 2006), and these native strains do not have pathogenicity in the traits we measured (Montenegro et al., 2006). Epigenetic drift towards lower titre during passage is also a poor explanation for reduced virulence, as titre effects such as these would be expected to also be reflected in reduced vertical transmission efficiency (not observed). Our favoured hypothesis is that the natural enemy protective mechanism observed in the native host misfires to damage the novel host, and selection acts to remove this misfire. Testing this hypothesis awaits future discovery of the mechanism of protection, in which toxin production has been implied (Hamilton et al., 2014), to establish whether its expression is induced in novel host species.

In this study, we showed that negative effects of Spiroplasma on host fitness were completely negated after 17 generations in D. melanogaster. This provides empirical support for the hypothesis that costs of infections of symbionts rapidly decline in novel hosts following lateral transfer. However, the symbiont's vertical transmission efficiency tended to increase only slowly over time. Our data suggest that natural selection may fail to 'rescue' a novel symbiosis where both vertical transmission is reduced, and physiological cost incurred, on transfer to a new host. Further, we consider that the strains of Spiroplasma established here represent a good model system for studies on symbioses, and expect that further studies using these strains should provide great insights into the evolutionary histories of symbioses.

\section{DATA ARCHIVING}

Data have been deposited in Dryad, http://doi.org/10.5061/dryad.7hs24.

\section{CONFLICT OF INTEREST}

The authors declare no conflict of interest.

\section{ACKNOWLEDGEMENTS}

We thank Mr S Price for helping with microinjection and rearing flies, and for advice on our experiment procedure, respectively. The microinjection device was kindly provided by Professor N Wedell (University of Exeter, UK). Dr E Kasuya (Kyushu University, Japan) kindly provided advice on the data analyses. We also thank Dr A Yamawo (JSPS fellow, Kyushu University, Japan) and three anonymous referees for comments on the manuscript. This work was supported by JSPS KAKEN (09J05297 and 12J03363) to SN.

Brownlie JC, Johnson KN (2009). Symbiont-mediated protection in insect hosts. Trends Microbiol 17: 348-354.

Carrington LB, Hoffmann AA, Weeks AR (2010). Monitoring long-term evolutionary changes following Wolbachia introduction into a novel host: the Wolbachia popcorn infection in Drosophila simulans. Proc R Soc Lond B Biol Sci 277: 2059-2068.

Clancy DJ, Hoffmann AA (1997). Behavior of Wolbachia endosymbionts from Drosophila simulans in Drosophila serrata, a novel host. Am Nat 149: 975-988.

Counce SJ, Poulson DF (1962). Developmental effects of the sex-ratio agent in embryos of Drosophila willistoni. J Exp Zool 151: 17-31.
Duron O, Bouchon D, Boutin S, Bellamy L, Zhou LQ, Engelstädter J et al. (2008). The diversity of reproductive parasites among arthropods: Wolbachia do not walk alone. BMC Bio/ 6: 27.

Gasparich GE, Whitcomb RF, Dodge D, French FE, Glass J, Williamson DL (2004). The genus Spiroplasma and its non-helical descendants: phylogenetic classification, correlation with phenotype and roots of the Mycoplasma mycoides clade. Int J Syst Evol Microbiol 54: 893-918.

Haine ER (2008). Symbiont-mediated protection. Proc R Soc Lond B Biol Sci 275: 353-361.

Hamilton PT, Leong JS, Koop BF, Perlman SJ (2014). Transcriptional responses in a Drosophila defensive symbiosis. Mol Ecol 23: 1558-1570.

Haselkorn TS, Cockburn SN, Hamilton PT, Perlman SJ, Jaenike J (2013). Infectious adaptation: potential host range of a defensive endosymbiont in Drosophila. Evolution 67: 934-945.

Haselkorn TS, Markow TA, Moran NA (2009). Multiple introductions of the Spiroplasma bacterial endosymbiont into Drosophila. Mol Ecol 18: 1294-1305.

Herren JK, Paredes JC, Schüpfer F, Lemaitre B (2013). Vertical transmission of a Drosophila endosymbiont via cooption of the yolk transport and internalization machinery. MBio 4: 00532-12.

Hutchence KJ (2011). The evolutionary ecology of host-parasite interactions between Drosophila and Spiroplasma. Chapter 3: Phenotype and transmission efficiency of artificial and natural male-killing Spiroplasma infections in Drosophila melanogaster. PhD thesis. University of Liverpool, Liverpool, UK.

Hutchence KJ, Pade R, Swif HL, Bennet D, Hurst GDD (2011). Phenotype and transmission efficiency of artificial and natural male-killing of Spiroplasma infections in Drosophila melanogaster. J Invert Pathol 109: 243-247.

Jaenike J (2012). Population genetics of beneficial heritable symbionts. Trends Ecol Evol 27: 226-232.

Jaenike J, Polak M, Fiskin A, Helou M, Minhas M (2007). Interspecific transmission of endosymbiotic Spiroplasma by mites. Biol Lett 3: 23-25.

Jaenike J, Unckless R, Cockburn SN, Boelio LM, Perlman SJ (2010). Adaptation via symbiosis: recent spread of a Drosophila defensive symbiont. Science 329: 212-215.

Jiggins FM, Hurst GDD (2011). Microbiology. Rapid insect evolution by symbiont transfer. Science 332: 185-186

Kageyama D, Anbutsu H, Watada M, Hosokawa T, Shimada M, Fukatsu T (2006). Prevalence of a non-male-killing Spiroplasma in natural populations of Drosophila hydei. Appl Environ Microbiol 72: 6667-6673.

Mateos M, Castrezana SJ, Nankivell BJ, Estes AM, Markow TA, Moran NA (2006). Heritable endosymbionts of Drosophila. Genetics 174: 363-376.

McGraw EA, Merritt DJ, Droller JN, O'Neill SL (2002). Wolbachia density and virulence attenuation after transfer into a novel host. Proc Natl Acad Sci USA 99: 2918-2923.

Montenegro H, Petherwick AS, Hurst GDD, Klaczko LB (2006). Fitness effects of Wolbachia and Spiroplasma in Drosophila melanogaster. Genetica 127: 207-215.

Montenegro H, Solferini VN, Klaczko LB, Hurst GDD (2005). Male-killing Spiroplasma naturally infecting Drosophila melanogaster. Insect Mol Biol 14: 281-288.

Mouton L, Thierry M, Henri H, Baudin R, Gnankine O, Reynaud B et al. (2012). Evidence of diversity and recombination in Arsenophonus symbionts of the Bemisia tabaci species complex. BMC Microbiol 12: S10.

Osaka R, Watada M, Kageyama D, Nomra M (2013). Detection of Spiroplasma from the mite Macrocheles sp. (Acari: Macrochelidae) ectoparasitic to the fly Drosophila hydei (Diptera; Drosophilidae): a possible route of horizontal transmission? Symbiont 60: 79-84.

Pool JE, Wong A, Aquadro CF (2006). Finding of male-killing Spiroplasma infecting Drosophila melanogaster in Africa implies transatlantic migration of this endosymbiont. Heredity 97: 27-32.

Raychoudhury R, Baldo L, Oliveira DCSG, Werren JH (2009). Modes of acquisition of Wolbachia: horizontal transfer, hybrid introgression and co-divergence in the Nasonia species complex. Evolution 63: 165-183.

Rice WR (1989). Analyzing tables of statistical tests. Evolution 43: 223-225.

Russell JA, Moran NA (2005). Horizontal transfer of bacterial symbionts: Heritability and fitness effects in a novel aphid host. Appl Environ Microbiol 71: 7987-7994.

Sakaguchi B, Poulson DF (1963). Interspecific transfer of the "sex-ratio" condition from Drosophila willistoni to D. melanogaster. Genetics 48: 841-861.

SAS Institute (2009). JMP 8, SAS Institute Inc.: Cary, NC, USA.

Tinsley MC, Majerus MEN (2007). Small steps or giant leaps for male-killers? Phylogenetic constraints to male-killer host shifts. BMC Evol Biol 7: 238

Walsh PS, Metzger DA, Higuchi R (1991). Chelex 100 as a medium for simple extraction of DNA for PCR-based typing from forensic material. Biotechniques 10: 506-513.

Weeks AR, Turelli M, Harcombe WR, Reynolds KT, Hoffmann AA (2007). From parasite to mutualist: rapid evolution of Wolbachia in natural populations of Drosophila. PLoS Biol 5: 99-1005.

Williamson DL, Poulson DF. (1979). Sex-ratio organisms (Spiroplasmas) of Drosophila. In: Whitcomb RF, Tully JG (eds) The Mycoplasmas vol. 3, Academic Press: New York, NY, USA. pp 175-208.

Williamson DL, Sakaguchi B, Hackett KJ, Whitcomb RF, Tully JG, Carle P et al. (1999). Spiroplasma poulsonii sp. nov., a new species associated with male-lethality in Drosophila willistoni, a neotropical species of fruit fly. Int J Syst Bacteriol 49: 611-618.

Xie JL, Vilchez I, Mateos M (2010). Spiroplasma bacteria enhance survival of Drosophila hydei attacked by the parasitic wasp Leptopilina heterotoma. PLOS ONE 5: 1-7.

Zug R, Koehncke A, Hammerstein P (2012). Epidemiology in evolutionary time: the case of Wolbachia horizontal transmission between arthropod host species. J Evol Biol 25: 2149-2160. 